\title{
LA APERTURA Y DESBLOQUEO DE LAS LISTAS ELECTORALES EN LAS COMUNIDADES AUTÓNOMAS. ¿ES POSIBLE? ${ }^{1}$
}

\author{
Andrés Iván Dueñas Castrillo \\ Profesor de Derecho Constitucional, \\ Universidad de Valladolid
}

Cómo citar este artículo / Citation: Dueñas Castrillo, A. I. (2021). La apertura y desbloqueo de las listas electorales en las Comunidades Autónomas. ¿Es posible? Palacios Romeo, F. y Cebrián Zazurca, E. (coords.) Elección y representación: una conjunción compleja. Perspectivas y problemas de los regímenes electorales en España, Colección Obras colectivas, Fundación Manuel Giménez Abad, Zaragoza.

DOI: https://doi.org/10.47919/FMGA.OC21.0306

SUMARIO: I. INTRODUCCIÓN - II. BREVE APROXIMACIÓN AL SISTEMA ELECTORAL DE LAS COMUNIDADES AUTÓNOMAS - III. LA POSIBILIDAD DE APERTURA Y EL DESBLOQUEO DE LAS LISTAS EN LAS COMUNIDADES AUTÓNOMAS - IV. CONCLUSIONES - V. BIBLIOGRAFÍA

\section{INTRODUCCIÓN}

El sistema electoral de las Comunidades Autónomas es un tema que ha sido tratado de diversas maneras por la doctrina (Oliver Araujo, 2011; Gavara de Cara, 2007; Gálvez Muñoz, 2009). Pero en los últimos años se han realizado cambios y propuestas que merece la pena señalar. Algunas han ido encaminados a mejorar la proporcionalidad de sus sistemas, a través de medidas que han afectado a la circunscripción electoral o que pretendían modificar la fórmula electoral (Fernández Esquer y Dueñas Castrillo, 2018:

\footnotetext{
${ }^{1}$ Investigación cofinanciada por el Fondo Social Europeo.

Trabajo realizado en el marco del Proyecto de Investigación DER2016-75993-P, sobre «España ante Europa: retos nacionales en materia de derechos humanos», que se desarrolla entre el 30 de diciembre de 2016 y el 29 de diciembre de 2020.
} 
63-65). Y otras propuestas han pretendido mejorar la participación, a través de la regulación de las primarias (Dueñas Castrillo, 2017: 18-27) o la apertura y el desbloqueo de listas.

Este capítulo pretende adentrarse en la última de las propuestas que se acaba de mencionar: la posibilidad de la apertura y el desbloqueo de listas. Para ello, se hará una primera incursión en el sistema electoral de las Comunidades Autónomas y qué posibilidades tienen de diferenciación. Posteriormente, se tratará de dar respuesta a si es posible una regulación así en el ámbito autonómico y qué implicaciones podría tener este cambio en el diseño de las listas electorales. Todo ello teniendo en cuenta las propuestas que han tenido lugar en las Comunidades Autónomas.

\section{BREVE APROXIMACIÓN AL SISTEMA ELECTORAL DE LAS COMUNIDADES AUTÓNOMAS}

Al sistema electoral se le ha definido de diversas maneras, entre otras, como "el conjunto de reglas y procedimientos conforme a los cuales se convocan y celebran las elecciones, se asignan los escaños a tenor de los votos obtenidos por las candidaturas y se resuelven los recursos a que todo este proceso diere lugar" (Torres del Moral, 2009: 207) o que "rigen el proceso electoral por el que las preferencias de los ciudadanos se articulan en votos y estos votos son traducidos en una distribución de autoridad gubernamental, normalmente escaños parlamentarios, entre los distintos partidos políticos" (Rae, 1977: 41). Es decir, cuando hablamos de sistema electoral nos referimos a "la totalidad orgánica de las distintas normas jurídicas, de las técnicas y procedimientos que se aplican al proceso, desde la apertura de las elecciones hasta la proclamación de los candidatos" (Schpeis, 1955: XXI).

En relación con lo anterior se puede afirmar que las elecciones son pieza fundamental e imprescindible de cualquier sistema constitucional democrático (Garrorena Morales, 2008: 21) y un requisito legitimador del poder político (Oliver Araujo, 2001: 23 y ss.). Como ya se sabe, las bases del sistema electoral español se establecieron en la Ley para la Reforma Política y el Real Decreto-Ley sobre normas electorales, de 18 de marzo de 1977, luego confirmadas por el artículo 68 CE y la Ley Orgánica de Régimen Electoral 
General (en adelante, LOREG). Pero antes de que esta se promulgara ya se había arraigado en los Estatutos de Autonomía y en la jurisprudencia del Tribunal Constitucional (Presno Linera, 2007: 105). La opción que defendió el constituyente fue la de optar por un sistema electoral que otorgase peso a los partidos y estabilidad al propio sistema de partidos. Por eso mismo se eligió el sistema de listas cerrado y bloqueado, a lo que se unen otras motivaciones: (i) se pretendía dar poder a los órganos directivos de los partidos, (ii) se alejaba al ciudadano del elegido (Tudela Aranda, 2018: 90) y (iii) se quería huir de la negativa experiencia del sistema mayoritario de listas abiertas utilizado en la II República (Fernández Esquer, 2018: 196).

Los Estatutos de Autonomía de las primeras Comunidades Autónomas, a las que hace mención el artículo $151 \mathrm{CE}$, se vieron influenciados por la normativa electoral estatal, hasta tal punto que alguna de las primeras redacciones de los Estatutos de Autonomía hacía remisiones explícitas de que su sistema electoral sería el mismo que rigiese para el Congreso de los Diputados (Presno Linera, 2007: 107). Ello ha traído consigo una fuerte homogeneidad entre los sistemas electorales autonómicos, tanto con el estatal, como entre sí (Gavara de Cara, 2007).

Varios son los factores que la doctrina ha apuntado como razones que han tendido hacia dicha homogeneidad. En primer lugar, históricas, dado que todas las Comunidades Autónomas hicieron suyo el modelo establecido en el artículo 152.1 CE, aunque no estuvieran obligadas a ello, y que posteriormente el Tribunal Constitucional ha establecido como canon de constitucionalidad en la sentencia 225/1998. También se ha de tener en cuenta el importante peso que tuvieron los Acuerdos Autonómicos de 1981 en la configuración del sistema electoral de las Comunidades.

En segundo lugar, competenciales. Hay que señalar que se ha hecho una interpretación extensiva de lo que se entiende por régimen electoral general, limitando y restringiendo la capacidad de las Asambleas Legislativas autonómicas. Aunque el artículo 148.1.1 a CE establezca que las Comunidades tienen competencia para la organización de sus instituciones de autogobierno (como es el régimen electoral de su Parlamento), estas atribuciones se han restringido por la vía de la disposición adicional 1a.2 de la LOREG y por la interpretación del Tribunal Constitucional (Gavara de Cara, 2007: 21). Es decir, el legislador autonómico se encuentra restringido. Pero, en cualquier caso, los 
Estatutos de Autonomía contienen importantes normas que afectan al sistema electoral, lo que ha llevado a que la doctrina los caracterice como códigos electorales autonómicos (Presno Linera, 2007: 143; y Biglino Campos, 2009: 21).

En tercer lugar se puede mencionar al Derecho comparado. En otros Estados compuestos también existe un cierto mimetismo entre el régimen electoral estatal y el de los Estados o Regiones o entre los propios entes territoriales. Si observamos la regulación alemana, el artículo 28.1 de la Ley Fundamental de Bonn establece el llamado principio u obligación de homogeneidad electoral, que significa que todas las elecciones, ya sean en el ámbito del Bundestag o de los Parlamentos de los Länder, han de garantizar los principios de sufragio universal, directo, libre, igual y secreto, lo que supone "una concreción del principio democrático y una garantía de la unidad de legitimación democrática, de forma que los mismos principios electorales son aplicables a cualquier proceso electoral, pero constituye un factor limitativo de la competencia en materia electoral de los Länder" (Gavara de Cara y Vallés Vives, 2007: 27). Pero también es cierto que en este país existe una variedad en los distintos componentes de los sistemas electorales de los Länder, lo cual "dibuja un panorama de relativa creatividad institucional" (Fernández Esquer, 2019: 110).

Otro ejemplo es Italia donde, aunque el artículo 122.1 de su Constitución (en adelante, $\mathrm{Cl}$ ) establece que la ley electoral regional debe ser un acto normativo autónomo, se ha restringido la capacidad de actuación del legislador regional. Tal y como sucede en el caso español, las Regiones tienen competencia para regular sus instituciones de autogobierno (el artículo $123 \mathrm{Cl}$ usa la expresión "forma de gobierno regional"), pero la Corte Constitucional ha limitado dicha competencia, como ha ocurrido en España con la STC 225/1998. Tras la reforma constitucional dispuesta para las Regiones ordinarias a través de la Ley Constitucional 1/1999, se ha establecido para dichas Regiones la elección directa del Presidente del Consiglio Regionale de manera transitoria, pudiendo elegir otra forma de elección los Estatutos, aunque finalmente todas han adoptado esta fórmula. La Corte Constitucional italiana ha tenido ocasión de pronunciarse al respecto y ha limitado la competencia del estatuyente y el legislador regional. Concretamente, ha dicho que las leyes regionales electorales tienen como fin dotar a la forma de gobierno regional de instrumentos adecuados de equilibrio y funcionamiento desde el momento de la 
constitución de los órganos de las Regiones (sentencia 196/2003) y que los Estatutos sólo pueden adoptar la decisión básica de la elección directa por sufragio universal del Presidente de la Junta Regional y lo demás debe ser regulado por ley regional (sentencia 2/2004).

Por tanto, como sucede en España, en Italia, a pesar de que se otorgue una competencia para la regulación de su propio sistema electoral a las Regiones ordinarias, esta se ha visto limitada a través de la legislación estatal y de la interpretación de la Corte Constitucional.

Se puede observar, por tanto, que en algunos Estados compuestos los regímenes electorales tienen una cierta homogeneidad, debido a la similitud de las formas de gobierno a nivel estatal-federal y territorial, la semejanza de criterios usados para la representación política o la permanencia temporal y estable de la normativa (Gavara de Cara y Vallés Vives, 2007: 418). Hay autores que piensan que este mimetismo no es malo, dado que, en el caso español, "a pesar de que evidencia cierta falta de creatividad por parte de los legisladores autonómicos, permite reconocer que existen, en nuestro ordenamiento, algunos principios comunes de Derecho electoral, hecho que facilita el conocimiento, la interpretación y la aplicación de las normas jurídicas". Es decir, que la autonomía "puede ser diversidad, pero no tiene que serlo siempre y por fuerza". Pero también es verdad que se ha constatado una "pereza normativa que aqueja al legislador electoral autonómico" (Presno Linera, 2007: 143), salvo algunas excepciones, como se verá a continuación, y que "el principio de homogeneidad no puede significar uniformidad (...), sino que se limita al control del respeto y la compatibilidad del Estatuto de Autonomía y el desarrollo normativo con los principios establecidos en el artículo 152.1 CE" (Gavara de Cara, 2007: 34-35).

Sin embargo, los principios políticos que hicieron surgir el sistema electoral español, trasladados posteriormente a las Comunidades Autónomas en su práctica totalidad, parecen estar en crisis. $Y$ es que "también al statu quo de los sistemas electorales, y a determinados aspectos del régimen electoral autonómico, les llega un punto de inflexión con la devastadora crisis económica que arrasó con la confianza de la ciudadanía en las instituciones representativas y de gobierno" (García Mahamut, 2018: 188). Ya en el Informe del Consejo de Estado de 2009 sobre las propuestas de modificación del 
Régimen Electoral Común se hacían enmiendas encaminadas a mejorar nuestro sistema en este sentido (Soriano Moreno, 2018: 80).

Pero actualmente las Comunidades Autónomas se han puesto a la vanguardia de los cambios encaminados a mejorar el sistema electoral que, en algunos aspectos, las han diferenciado entre sí y con el sistema del Congreso de los Diputados. A pesar de la homogeneidad a la que se ha hecho referencia, el estatuyente y el legislador autonómico tienen margen de maniobra para poder singularizarse en algunos aspectos.

Aunque el legislador autonómico tenga un estrecho margen de maniobra, la competencia de las Comunidades Autónomas del artículo 148.1.1 a CE no es una excepción al régimen electoral general, sino que es una competencia que se les atribuye de manera directa por la Constitución y sus Estatutos (Presno Linera, 2007: 130). La mayoría de Comunidades Autónomas no han asumido en sus Estatutos la materia electoral como un título competencial específico, sino que lo han deducido de su potestad de autoorganización. Actualmente tan solo lo reconocen como título competencial en sus Estatutos Navarra (artículo 49.1), País Vasco (artículo 10.3) -fueron las primeras en hacerlo- o Andalucía (artículo 46).

El legislador autonómico puede entrar a normar aspectos tan relevantes como la fórmula electoral proporcional, la circunscripción, el número de escaños de su Parlamento, el sistema de listas cerradas y bloqueadas, las barreras electorales, el procedimiento electoral, la Administración electoral, la forma de emisión del voto, la figuras de los apoderados e interventores, las funciones de los representantes de las candidaturas, el funcionamiento de las mesas, los procedimientos de escrutinio, las subvenciones electorales 0 aspectos importantes de la campaña electoral como pueden ser el papel que corresponde a los poderes públicos y los criterios para distribuir los espacios gratuitos en los medios de comunicación pública.

Por tanto, las Comunidades Autónomas tienen posibilidades de regular la apertura y el desbloqueo de las listas electorales. De esto tratan las siguientes líneas. 


\section{LA POSIBILIDAD DE LA APERTURA Y EL DESBLOQUEO DE LISTAS EN LAS COMUNIDADES AUTÓNOMAS}

Ya se ha explicado al inicio de este capítulo los motivos por los cuales se eligió en España un modelo de listas cerrado y bloqueado. $Y$ es que es uno de los factores del grado de protagonismo que tienen los partidos políticos en el sistema político (Carrasco Durán, 2018: 2). Actualmente en España, debido también a la falta de democracia interna, los partidos cuentan con un peso muy importante a la hora de la selección de candidatos lo que ha provocado que parte de la doctrina afirme que es el partido político quien elige a los representantes y la ciudadanía únicamente ratifica su elección (Carrasco Durán, 2018: 2). Las críticas de la doctrina a este sistema de listas apuntan en tres direcciones: son poco democráticas porque se priva a los electores de elegir entre los candidatos, fortalecen las posiciones de poder de las burocracias partidistas y son causa de la despersonalización de la relación entre representantes y representados y su distanciamiento (Santamaría Ossorio, 1994: 117).

Si acudimos a otros países, como Italia, su Corte Constitucional ha dicho en la sentencia 1/2014 que las listas bloqueadas privan a los electores de cualquier margen de elección de sus propios representantes, que sería totalmente dejada a los partidos (FJ 5.1). También la Sala Constitucional de la Corte Suprema de Justicia de El Salvador, en 2009 y 2014, ha interpretado que ese modelo vulneraba el carácter libre del voto (Carrasco Durán, 2018: 7 y 25).

En el Derecho comparado se puede observar cómo los países que han adoptado listas cerradas y bloqueadas provienen de regímenes dictatoriales anteriores o en los que se ha querido esquivar los problemas causados en el pasado por una excesiva fragmentación política. En Europa existe este modelo de listas en Portugal, Serbia, Albania, Macedonia, Montenegro, Hungría, Turquía, Andorra, Moldavia, Ucrania, Kazajistán, Georgia, Alemania y Rusia (Carrasco Durán, 2018: 3).

A diferencia de lo que ocurrió en la Transición y en los primeros años de democracia, hoy en día "los partidos políticos españoles son vistos como organizaciones con propensión a extender su poder institucional más allá de lo que legítimamente les corresponde" (Fernández Esquer, 2019: 149). Es decir que "las estructuras organizativas de los partidos políticos han acumulado tal 
cantidad de poder que (...) las listas cerradas y bloqueadas ya no resultan admisibles desde la perspectiva de una democracia avanzada" (Oliver Araujo, 2011: 287). El propio Consejo de Estado, en su informe de 2009, dijo que era necesario que se hicieran reformas "encaminadas a logar alguna modalidad de votación más flexible" (Consejo de Estado, 2009: 154).

Las listas abiertas se pueden definir como aquel sistema "en que el elector puede escoger entre los candidatos incluidos en un orden convencional o bien combinar candidatos de listas distintas, la fórmula conocida como panachage" (Almagro Castro, 2018: 119). En ellas "el elector tiene una libertad total en esa segunda etapa que corresponde al acto formal de votación" (Fernández Esquer y Sierra-Rodríguez, 2018: 61). Pero hay que distinguir entre las listas desbloqueadas y abiertas. En las primeras se permite a los electores "mostrar su preferencia por algún o algunos candidatos de una lista electoral concreta y, en casos excepcionales, dar un voto de rechazo a algún candidato de la lista", mientras que en las segundas "el elector puede elegir candidatos de distintas listas electorales e, incluso, dar varios votos a un candidato o mostrar su rechazo a alguno de ellos" (Carrasco Durán, 2018: 9 y 10). Los tipos de listas desbloqueadas pueden ser, a su vez, muy diferentes (Fernández Esquer, 2019: 143-145).

Entre las ventajas asociadas a una mayor personalización del voto que comportaría un sistema de listas abiertas y desbloqueadas se han apuntado varias. En primer lugar, que el elector decidiría no solo cuantos diputados le corresponden a cada partido, también qué candidatos ocuparán esos escaños, un menor poder para las cúpulas partidarias, mejorar la relación entre representantes y representados (Fernández Esquer, 2019: 145-159), obligar a los partidos a tomar en cuenta más intensamente las preferencias de los electores (Carrasco Durán, 2018: 31) o un mayor acercamiento a los electores, lo que supone, en definitiva, mayor responsabilidad de los representantes (Garrote de Marcos, 2017: 401) y de esta manera se podrían evitar casos como los conocidos diputados "paracaidistas". Se podría hacer un símil con un restaurante, donde "las listas cerradas y bloqueadas serían el menú del día, mientras que las listas abiertas sería pedir a la carta" (Biglino Campos, 2020: 11).

Entre las críticas, se puede argumentar que se perjudica la cohesión y unidad de los partidos (Fernández Vivas, 2020: 391). Ello es así porque "los 
candidatos pueden tener incentivos para competir con sus compañeros de partido" (Fernández Esquer, 2019: 150), algo que puede resultar paradójico si se tiene en cuenta que la ciudadanía española cuestiona el poder oligárquico de los partidos pero rechaza que éstos se presenten divididos (Almagro Castro, 2018: 127). Pero, en algunos países como Eslovaquia, sucede lo contrario. Alí los partidos incluyen en sus listas a diputados díscolos que en muchas ocasiones se han saltado la disciplina de partido, que normalmente son aquellos que han resultado elegidos por el voto preferencial, buscando una reputación personal de los parlamentarios que beneficie al partido (Garrote de Marcos 2017: 391).

Ya se ha visto que las Comunidades Autónomas pueden incidir en sus modelos de listas. Durante la legislatura 2015-2019 se realizaron varias propuestas para abrir y desbloquear las listas en Asturias $^{2}$, Valencia $^{3}$, La Rioja ${ }^{4}$ o Cataluña ${ }^{5}$, con diferentes grados de concreción en la medida. Ninguna de ellas fue finalmente aprobada.

En el caso de La Rioja tan sólo se dice en el artículo 15.2 de la proposición de ley que "en las elecciones a la Diputación General de La Rioja, el sistema electoral será el de listas abiertas y desbloqueadas, pudiendo cada votante dentro de una misma papeleta, señalar a un número máximo de Diputados igual al que le corresponda en su circunscripción". Es la única que también propone una apertura de las listas, todas las demás tan sólo un desbloqueo.

En la propuesta catalana los electores podrían otorgar un voto preferencial a cualquier candidato. Lo harían a entre 1 y 25 candidatos en la circunscripción de Barcelona y a entre 1 y 8 en las demás circunscripciones (artículo III-7 de la propuesta). Antes, en 2010 , en Cataluña se había registrado una ILP que pretendía "la incorporación de las listas desbloqueadas con voto preferencial y la representación equilibrada en cada tramo de 5 nombres, siguiendo la distribución 40/60\%” (Queralt Jiménez, 2017: 420).

\footnotetext{
${ }^{2}$ Boletín Oficial de la Junta General del Principado de Asturias de 3 de febrero de 2016, oㅡ 19.1, serie A.

${ }^{3}$ Boletín Oficial de las Cortes Valencianas de 5 de abril de 2018, no 262, pp. 44037 y ss.

${ }^{4}$ Boletín Oficial del Parlamento de La Rioja de 3 de noviembre de 2015, no 10, serie A, pp. 54 y ss.

${ }^{5}$ Boletín Oficial del Parlamento de Cataluña de 5 de mayo de 2016, № 122, pp. 9 y ss.
} 
Asturias probablemente constituya la propuesta más elaborada ya que la que se ha hecho en la legislatura 2015-19 viene de otra que se realizó en el anterior período legislativo (García Mahamut, 2018: 199). En la última se establecía un desbloqueo de las listas de tal forma que el votante podía disponer de un voto preferencial no acumulable a un máximo de nueve candidatos dentro de cada lista. Los que superasen el $5 \%$ sobre "el conjunto de sufragios recibidos por el partido en la circunscripción de que se trate, pasarían a ser elegidos de manera preferente", sin que pudiera ver alterada su posición el cabeza de lista porque previamente habría sido elegido por un proceso de primarias obligatorio (Fernández Esquer, 2019: 30-31). Esta propuesta, de nuevo, no se llegó a aprobar debido a que los Grupos Parlamentarios no retomaron el debate después de decidir aparcarlo a la espera de una posible modificación de la regulación estatal ${ }^{6}$.

Por último, en la Comunidad Valenciana se proponía un voto preferencial con la posibilidad de marcar opcionalmente a un máximo de tres personas, con un blindaje respecto a quien ocupase la primera posición de la lista. Además se estipulaba una solución al problema del equilibrio de sexos al que se ha hecho alusión antes, de tal manera que "el voto preferencial podría alterar la posición ocupada por los candidatos en la papeleta a los efectos de la asignación de escaños, pero solo respecto a los candidatos de su mismo sexo" (Fernández Esquer y Sierra-Rodríguez, 2018: 37-38).

\section{CONCLUSIONES}

El constituyente español y, posteriormente, el legislador orgánico, confirmaron el modelo electoral establecido en 1977. Esto es, un sistema proporcional con sesgos mayoritarios con un sistema de listas cerradas y bloqueadas, que buscaba otorgar protagonismo y fortalecer a los partidos políticos.

Posteriormente, las Comunidades Autónomas optaron por hacer suyo casi en su totalidad el sistema electoral para el Congreso de los Diputados. Ello se ha debido a diferentes causas, entre las que se pueden destacar los Acuerdos Autonómicos de 1981, la posterior elevación a canon de constitucionalidad para

$6 \quad$ https://www.Ine.es/asturias/2017/05/31/junta-aparca-indefinidamente-propuestareforma/2113017.html 
todas las Comunidades del sistema institucional del artículo 152.1 CE y la intervención del legislador orgánico y la propia jurisprudencia constitucional, que han dejado poco margen al legislador autonómico para innovar en su normativa. Ello ha traído como consecuencia una fuerte homogeneidad entre los sistemas electorales autonómicos, como sucede en otros países de nuestro entorno que cuentan con una descentralización territorial.

Pero también es cierto que las Comunidades Autónomas tienen cierto espacio para diferenciar sus sistemas electorales. Nuestros entes subestatales autonómicos han realizado propuestas de cambio y modificaciones en sus normas electorales para tratar de hacer frente a las críticas provenientes de la sociedad, la doctrina o la propia política. Aunque no se hayan llevado a término, como se ha podido ver en las páginas precedentes, ha habido interesantes proposiciones referidas al desbloqueo de las listas. Ello otorgaría más protagonismo a los ciudadanos y podría evitar que la cúpula de los partidos políticos eligieran a los candidatos electorales, que luego los representados se limitan a ratificar con la emisión de su voto.

En cualquier caso, es pronto para aventurar una conclusión acerca de estas medidas. Ninguna de ellas se ha llegado a aprobar. Pero se podría decir que las Comunidades Autónomas son el espacio idóneo para ponerlas en práctica. El tamaño de sus Parlamentos y la cercanía de los representantes con los representados, entre otras razones, las hacen idóneas para aventurarse a explorar fórmulas que, en el caso de ser exitosas, se podrían extrapolar a otros ámbitos como el estatal y ayudar a mejorar nuestra democracia.

\section{BIBLIOGRAFÍA}

- ALMAGRO CASTRO, D. (2018). La apertura de las listas electorales: ¿un primer paso hacia la superación de la crisis de la representatividad española? Revista Española de Derecho Constitucional, 112.

- BIGLINO CAMPOS, P. (2009). La legislación electoral estatal y el margen de apreciación del legislador autonómico. En Gálvez Muñoz, L. A., (dir.), El Derecho electoral de las Comunidades Autónomas. Revisión y mejora, Madrid: Centro de Estudios Políticos y Constitucionales. 
- (2020). La reivindicación del Parlamento en la época del populismo. ¿Cómo regenerar su propia legitimación? Artículo pendiente de publicación. Manuscrito cedido por la autora.

- CARRASCO DURÁN, M. (2018). Listas electorales, ¿bloqueadas o desbloqueadas? ¿Cerradas o abiertas? Revista General de Derecho Constitucional, ํㅡe extraordinario.

- Consejo de Estado (2009). Informe sobre las propuestas de modificación del Régimen Electoral.

- DUEÑAS CASTRILLO, A. I. (2017). La investidura de los Presidentes autonómicos y primarias en los partidos políticos: fortalecimiento del presidencialismo parlamentario autonómico. Revista de Estudios Jurídicos, 17.

- FERNÁNDEZ ESQUER, C. (2018), El sistema electoral del Congreso de los Diputados (1977-2016): génesis, rendimientos y demandas de reforma. En Dueñas Castrillo, A. I.; Fernández Cañueto, D.; y Moreno González, G. (coords.), Juventud y Constitución. Un estudio de la Constitución española por los jóvenes en su cuarenta aniversario, Zaragoza: Fundación Manuel Giménez Abad.

- FERNÁNDEZ ESQUER, C. (2019), Los sistemas electorales de los Länder alemanes: panorámica general y tendencias de reforma. Revista Española de Derecho Constitucional, 117

- (2019). Las listas desbloqueadas en España: ¿regeneración democrática o espejismo institucionalista? En, Apuntes Electorales, 61.

- FERNÁNDEZ ESQUER, C. y DUEÑAS CASTRILLO, A. I. (2018). Sistema electoral y sistema de partidos de Castilla y León: pasado, presente y futuro. Revista Jurídica de Castilla y León, 45.

- FERNÁNDEZ ESQUER, C. y SIERRA-RODRÍGUEZ, J. (2018). El voto preferencial en las Comunidades Autónomas: propuestas y condicionantes jurídicos. Revista d'Estudis Autonomics i Federals, 29. 
- FERNÁNDEZ VIVAS, Y. (2020). El sistema electoral en Alemania a examen. Teoría y Realidad Constitucional, 45.

- GÁlVEZ MUÑOZ, L. A. (dir.) (2009). El Derecho electoral de las Comunidades Autónomas. Revisión y mejora. Madrid: Centro de Estudios Políticos y Constitucionales.

- GARCÍA MAHAMUT, R. (2018). La reforma de los sistemas electorales autonómicos tras las elecciones de 24 de mayo de 2015: vectores políticos y jurídicos. Teoría y Realidad Constitucional, 41.

- GARRORENA MORALES, A. (2008). Reforma y rigidez de la ley electoral en las Comunidades Autónomas. En Biglino Campos, P. (dir.), Nuevas expectativas democráticas y elecciones, Madrid: Iustel.

- GARROTE DE MARCOS, M. (2017). Listas desbloqueadas en los sistemas electorales autonómicos: ¿Es necesario, posible y probable? En PérezMoneo, M. y Vintró Castells, J., Participación política: deliberación y representación en las Comunidades Autónomas, Madrid: Congreso de los Diputados.

- GAVARA DE CARA, J. C. (2007). La homogeneidad de los regímenes electorales autonómicos, Madrid: Centro de Estudios Políticos y Constitucionales-Fundación Manuel Giménez Abad.

- GAVARA DE CARA, J.C. y VALLÈS VIVES, F. (2007). Los regímenes electorales territoriales en los Estados compuestos. Alemania, Estados Unidos e Italia, Madrid: Congreso de los Diputados.

- OliVER ARAUJO, J. (2011). Los sistemas electorales autonómicos, Barcelona: Institut d'Estudis Autonòmics.

- PRESNO LINERA, M. A. (2007). Nuevos Estatutos de Autonomía con viejos sistemas electorales. Revista d'estudis autonomics i federals, 5.

- QUERALT JIMÉNEZ, A. (2017). Las listas desbloqueadas en el sistema electoral catalán: ¿mucho ruido y pocas nueces? En Pérez-Moneo Agapito, M. y Vintró Castells, J., Participación política: deliberación y representación en las Comunidades Autónomas, Madrid: Congreso de los Diputados. 
- RAE, D.W (1977). Leyes electorales y sistemas de partidos políticos, Madrid: CITEP, Madrid.

- SANTAMARÍA OSSORIO, J. (1994). Listas cerradas, abiertas y entreabiertas. En La reforma del régimen electoral, Madrid: Centro de Estudios Constitucionales.

- SCHPEIS, G. (1955). I sistema elettorali: teoria, tecnica legislazioni positive, Empoli (Italia): Caparrini.

- SORIANO MORENO, S. (2018). El sistema electoral del Congreso de los Diputados: análisis, proporcionalidad y propuestas de mejora. En Palacios Romeo. F. y Cebrián Zazurca, E. (eds.), Elegir y cómo elegir. Retos y urgencias del régimen electoral en España, Zaragoza: Fundación Manuel Giménez Abad.

- TORRES DEL MORAL, A. (2009). Sistemas electorales y sistemas de partidos en las Comunidades Autónomas. En Gálvez Muñoz, L.A. (dir.). El Derecho electoral de las Comunidades Autónomas. Revisión y mejora, Madrid: Centro de Estudios Políticos y Constitucionales, Madrid.

- TUDELA ARANDA, J. (2018). Exigencias constitucionales del Estado de Derecho y del principio democrático en relación con el Estado autonómicoEn Tudela Aranda, J., Kölling, M. y Reviriego, F. (coords.), Calidad democrática y organización territorial, Madrid: Marcial Pons. 